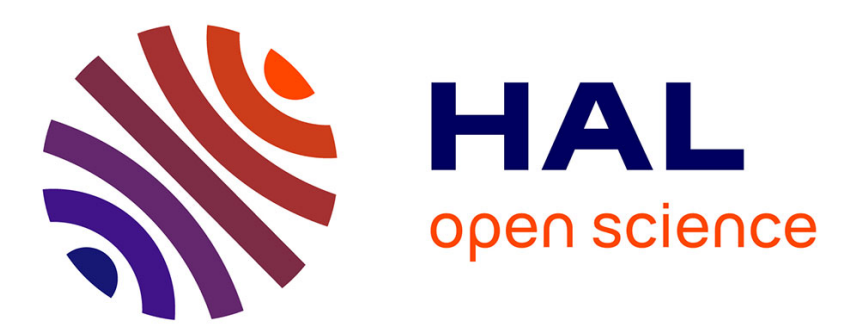

\title{
Compensation of loss to approach -1 effective index by gain in metal-dielectric stacks
}

\author{
J. Zhang, H. Jiang, B. Gralak, S. Enoch, G. Tayeb, M. Lequime
}

\section{To cite this version:}

J. Zhang, H. Jiang, B. Gralak, S. Enoch, G. Tayeb, et al.. Compensation of loss to approach -1 effective index by gain in metal-dielectric stacks. European Physical Journal: Applied Physics, 2008, 46 (3), pp.1-6. 10.1051/epjap:2008148 . hal-00480178

\section{HAL Id: hal-00480178 https://hal.science/hal-00480178}

Submitted on 3 May 2010

HAL is a multi-disciplinary open access archive for the deposit and dissemination of scientific research documents, whether they are published or not. The documents may come from teaching and research institutions in France or abroad, or from public or private research centers.
L'archive ouverte pluridisciplinaire HAL, est destinée au dépôt et à la diffusion de documents scientifiques de niveau recherche, publiés ou non, émanant des établissements d'enseignement et de recherche français ou étrangers, des laboratoires publics ou privés. 


\section{Compensation of loss to approach -1 effective index by gain in metal-dielectric stacks}

Jinlong Zhang ${ }^{1,2}$, Haitao Jiang ${ }^{1,3}$, Boris Gralak ${ }^{1}$, Stefan Enoch $^{1}$, Gérard Tayeb ${ }^{1}$, and Michel Lequime $^{1}$

${ }^{1}$ Institut Fresnel, CNRS, Aix-Marseille Université, Domaine Universitaire de Saint Jérôme, service 161, 13397 Marseille cedex 20

${ }^{2}$ State Key Laboratory of Modern Optical Instrumentation, Zhejiang University, Hangzhou, 310027 China

${ }^{3}$ Pohl Institute of Solid State Physics, Tongji University, Shanghai, 200092, China

jinlongzhang@zju.edu.cn

PACS: 42.25.-p, 78.66.Bz, 78.67.Pt 
Abstract: We propose a theoretical study of optimization of metal-dielectric multilayer in order to approach -1 effective refractive index for transverse magnetic waves and a wavelength in the visible. The absorption losses of metal appear to be a crucial factor that affects the effective properties of the multilayer. Taking advantage of the dispersion relation of Bloch modes, we show that the losses not only decrease the transmission of the stack, but also change the negatively refracted angle. Then, we propose that using a gain-providing semiconductor ( $\mathrm{GaN}$ ) may allow compensating for the losses in metal layers. In theory, the performances of the structure can be improved greatly when gain is involved. When considering finite thickness structures, and with appropriate thickness for the terminating layers, it is possible to obtain a high transmission of the structure. A near -1 effective index metal-dielectric stack with high transmission may pave the way to the realization of negative quasi-isotropic refraction in the visible or ultraviolet wavelength range. 


\section{Introduction}

Negative refraction was early suggested by V.G. Veselago in a theoretical study of left-handed (LH) materials whose permittivity and permeability are simultaneously negative [1]. In 2000, J. Pendry has shown that in addition to producing negative refraction effect, a LH material could be used to build a perfect lens that can overcome the diffraction limit [2]. Obviously, such a result has attracted a lot of attention from scientific community.

Although nature does not provide us with such a material, at least in optical range, nowadays negative refraction has been experimentally verified in some metamaterials [3, 4]. There are also many articles reporting theoretical and experimental study of negative refraction in photonic crystals (PCs) [5-7]. However it is still a formidable challenge to create metamaterials that have suitable properties in optical range of wavelength.

An alternative approach has been suggested: for H-polarization case a metallic layer may act as a superlens in the electrostatic limit [2]. Using such a simple structure super resolution has been confirmed by experimental results [8-10].

Recently, several groups have reported studies of negative refraction and sub-wavelength imaging using metal-dielectric multilayers [11-16]. In technology, one-dimensional (1D) stack are easy to fabricate and can achieve negative refraction in optical or even ultra-violet wavelength. Moreover, it has been shown that they could have better properties than a single metallic layer [11]. In practice, isotropic effective materials are highly desirable. A nearly -1 isotropic effective index for all angles of incidence near visible wavelength has been achieved using this 1D stack with proper design [16]. In Ref. [16], we chose a structure in which the ratio of the imaginary part to the real part of the permittivity of a metal/ dielectric is relatively low to decrease the deleterious effect of losses. In this paper, we consider another way to approach nearly -1 isotropic effective index by using a gain-providing material $(\mathrm{GaN})$ to compensate for the losses in metal layers.

\section{1D stack with lossless materials}

The 1D stack is schematically shown in Fig. 1. It is composed of alternating layers of dielectric and metal with permittivities $\varepsilon_{1,2}$ and thicknesses $d_{1,2}$, respectively. Both metal and dielectric are assumed nonmagnetic $\left(\mu_{1}=\mu_{2}=1\right)$. We suppose that the $Z$-axis is normal to each layer. A symmetric unit cell is used in the stack in order to follow the appropriate truncation leading to the optimized reflexion and transmission properties $[14,15]$.

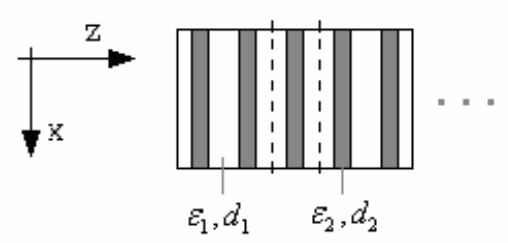

Fig.1. A 1D metal-dielectric stack. The dashed line shows the symmetric unit cell. 
First, for simplicity, we use a lossless Drude model to describe the dielectric function of the metal layers, $\varepsilon_{2}=1-\omega_{p}^{2} / \omega^{2}, \omega_{p}$ is the bulk plasma frequency of the metal. In the present paper, we only consider H-polarization case. Based on the analysis developed in Ref. [17], the dispersion relation of 1D stack is given by:

$$
\cos \left(K_{z} d\right)=\cos \left(\alpha_{1} d_{1}\right) \cos \left(\alpha_{2} d_{2}\right)-\frac{\alpha_{1}^{2} \varepsilon_{2}^{2}+\alpha_{2}^{2} \varepsilon_{1}^{2}}{2 \alpha_{1} \alpha_{2} \varepsilon_{1} \varepsilon_{2}} \sin \left(\alpha_{1} d_{1}\right) \sin \left(\alpha_{2} d_{2}\right)
$$

where $K_{z}$ is the Bloch wave vector, $d=d_{1}+d_{2}, \quad \alpha_{i}=\sqrt{k_{0}^{2} \varepsilon_{i} \mu_{i}-k_{x}^{2}},(i=1,2), \quad k_{x}$ is the parallel wave vector, and $k_{0}=\omega / c$ is the wave vector of light in free space. The existence of a propagating Bloch mode requires $\left|\cos \left(K_{z} d\right)\right| \leq 1$. After an optimization, we find a set of parameters $\left(\varepsilon_{1}=9, \omega_{p}=9.7 \mathrm{fs}^{-1}, d_{1}=30 \mathrm{~nm}\right.$, and $\left.d_{2}=36 \mathrm{~nm}\right)$ that can realize a nearly isotropic -1 effective index structure.

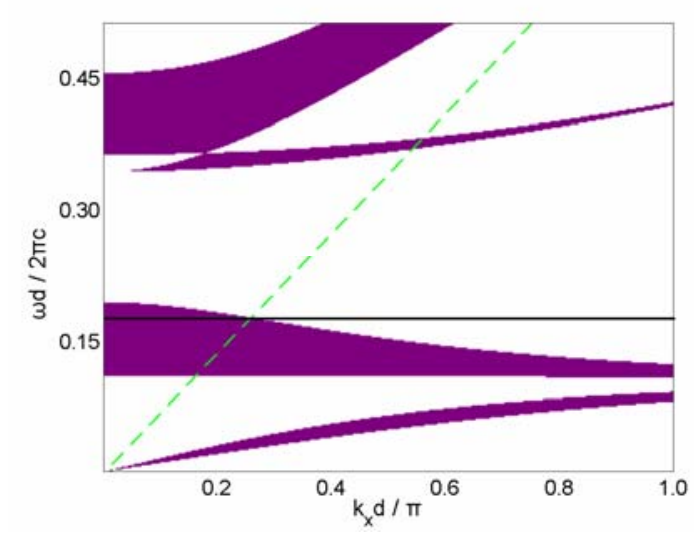

(a)

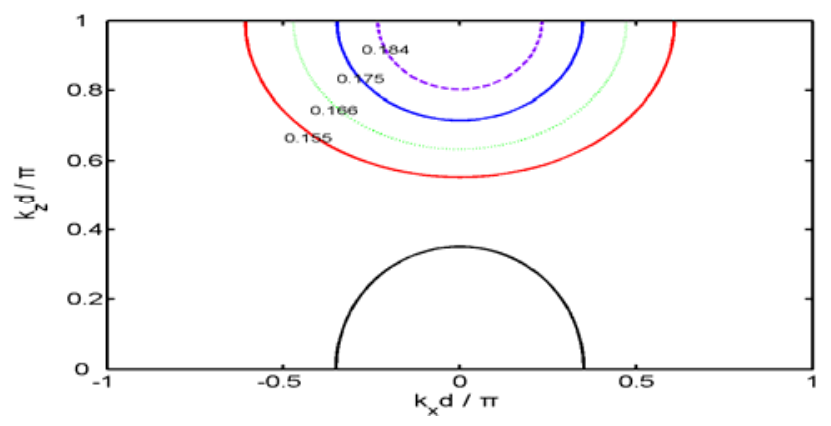

(b)

Fig.2. (a) Band structure of 1D stack. The purple zones represent the pass bands. The green dashed line represents the light line in free space. The black solid line indicates the frequency at which $n_{e f f}=-1 . \quad k_{p}=\omega_{p} / c$. (b) EFCs for the second band, the labels indicate the frequencies in unit of $2 \pi c / d$. The black curve is EFC of free space for $\omega=0.175 * 2 \pi c / d$. 
For this structure, we have computed the band structure and equal frequency contours (EFCs) shown in Fig.2 using transfer matrix formalism [5]. From Fig.2 (b), one sees that at frequency $\omega=0.175 * 2 \pi c / d$, the EFC is almost circular (indicating that the structure can be considered as a nearly isotropic medium) and $n_{\text {eff }}$ can be well defined at this frequency. Note that the circular EFC is not centered at the origin but on the point $\mathrm{M}\left(k_{x}=0, K_{z}=\pi / d\right)$ [see Fig. 2 (b)]. However, if the interface delimiting the structure is perpendicular to the z-axis, then this position of the EFC has no effect on the refractive properties of the structure. Also note that the EFC's radius decreases when the frequency increases, this indicates that the group velocity $\left(\mathbf{v}_{g}=\operatorname{grad}_{\mathbf{k}}(\omega)\right)$ will point towards the point $\mathbf{M}$ and thus negative refraction is expected. We found that at $\omega=0.175 * 2 \pi c / d \quad\left(\varepsilon_{2}=-2.8\right)$, the radius of EFC is almost equal to that of the EFC of the free space. Thus, the effective index $n_{\text {eff }}$ of this stack is nearly -1 at this frequency.

In order to analyze the negative refraction effect of this $1 \mathrm{D}$ stack, we calculated the field map when a Gaussian beam transmits a 1D structure with a finite thickness. Note that in the considered model, the Gaussian beam is limited only along one direction, thus the incident magnetic field is given by the following relation:

$$
H_{i y}=\int_{\infty}^{-\infty} d k_{x} \exp \left[i\left(k_{x} x+k_{i z} z\right)\right] \phi\left(k_{x}\right)
$$

where

$$
\phi\left(k_{x}\right)=\frac{g}{2 \sqrt{\pi}} \exp \left[-g^{2}\left(k_{x}-k_{i x}\right)^{2} / 4\right] .
$$

The incident beam is centered at $k_{i}=\hat{x} k_{i x}+\hat{z} k_{i z}=\hat{x} k_{0} \sin \theta_{i}+\hat{z} k_{0} \cos \theta_{i}, \theta_{i}$ is the incident angle, $\mathrm{g}$ represents the width of the waist. In the following simulation work, we choose $g=2 \lambda$.

As an example, we consider a 5-period stack (total thickness is $330 \mathrm{~nm}$, with $180 \mathrm{~nm}$ of metal) surrounded by free space. Figure 3 shows the time-averaged power density calculated using the transfer matrix method [18]. The Gaussian beam is incident from left with an angle of $45^{\circ}$. The beam is refracted at the interface with an angle of about $-43.6^{\circ}$. The little difference with the angle deduced from the dispersion relation $\left(-41.3^{\circ}\right)$ could be attributed to the fact that, first, we consider a finite thickness stack and, also, to the finite width of the Gaussian beam and thus its non-null angular width. In this case the transmittance is high in a wide range of incident angle (see Fig.8, for the lossless structure, i.e. the blue curve). 


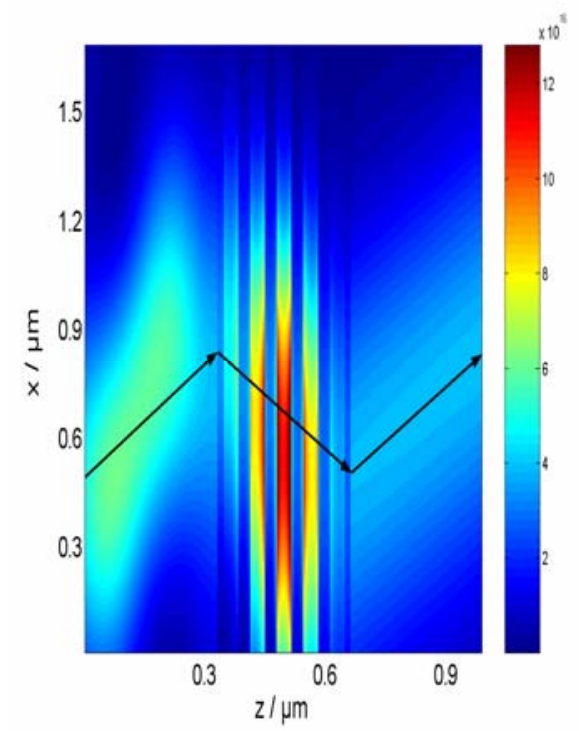

Fig.3. Time-averaged power density distribution for negative refraction of a Gaussian beam by a 5 -period stack (H polarization) with an angle of incidence: $45^{\circ}$. The arrow line in the map shows the locus of the maximum incident, refracted and transmitted fields. Arbitrary unit is used for the power density in all the maps.

We also performed a finite-difference-time-domain (FDTD) simulation for focusing by such a 5-period stack at $\omega=0.175 * 2 \pi c / d\left(\varepsilon_{2}=-2.8\right)$. A point source was placed at the left-hand side of the slab with a distance of $120 \mathrm{~nm}$ from the slab surface. Figure 4 shows a snapshot of the distribution of the real part of the magnetic field $\left(H_{y}\right)$, which clearly shows an image formed in the opposite side of the structure in air, the distance between the object and image point is $648 \mathrm{~nm}$, about twice of the slab width. This confirms that the stack can be considered as an effective homogeneous material whose effective index $n_{\text {eff }}$ is close to -1 . The full width at half maximum (FWHM) of image is $0.55 \lambda$. The fact that the resolution is not very good is due to the absence of evanescent waves in the imaging process but note that the structure has not been optimized with this aim.

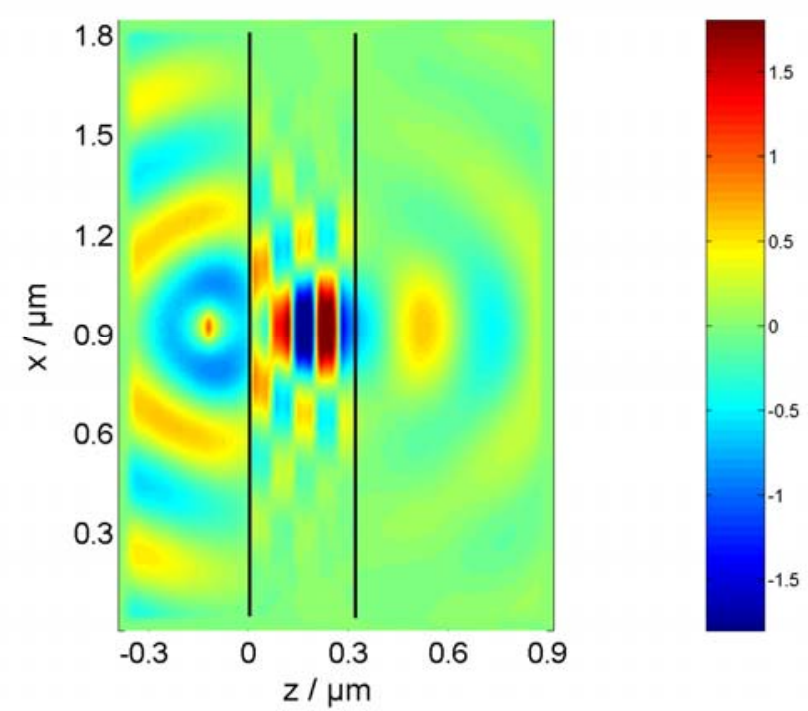

Fig.4. Focusing with a 5-period stack that is between the two black lines. Plotted here is a snapshot of the distribution of the real part of the magnetic field $\left(H_{y}\right)$. 


\section{Effects of the absorption losses on the negative refraction}

Taking into account realistic material parameters of metal, at the operating wavelength $(\lambda=376 \mathrm{~nm})$, the dielectric constants for silver is $\varepsilon_{2}=\varepsilon_{\text {siver }}=-2.8+0.3 i$ [19]. The dielectric layer is assumed to be lossless. We calculate the complex Bloch vector of $1 \mathrm{D}$ stack at $\lambda=376 \mathrm{~nm}$ in Fig. 5 . The real component of Bloch vector corresponds to the wave number (one can check that the average energy flow direction is given by $\mathbf{v}_{g}=\operatorname{grad}_{\text {real }(\mathbf{k})}(\omega)$ ), while the imaginary part determines the absorption $[20]$.

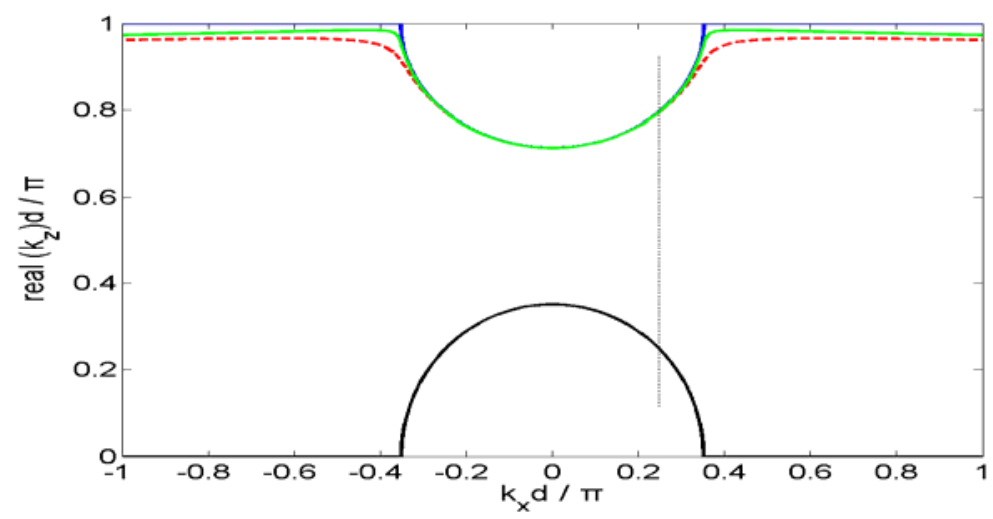

(a)

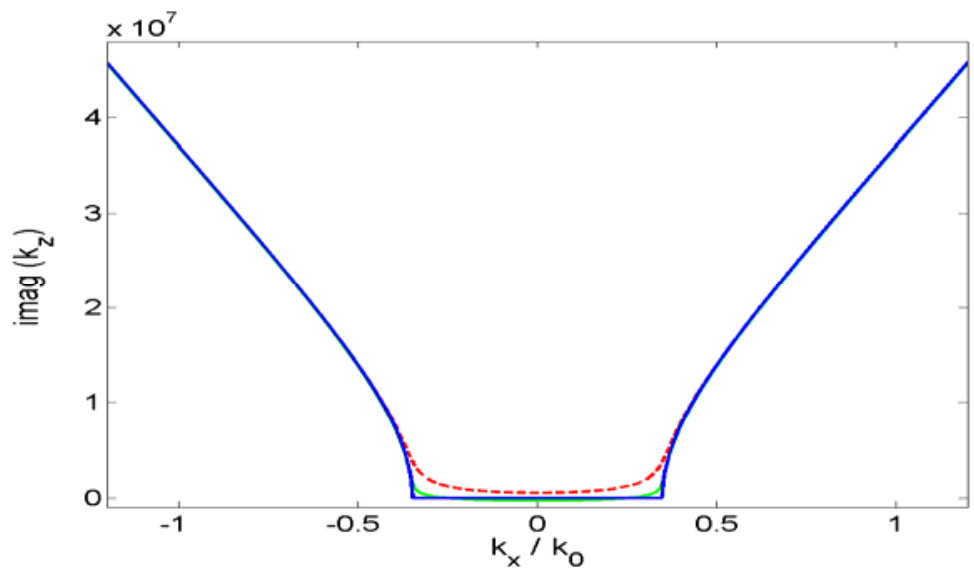

(b)

Fig.5. The red dashed curve is the real component of the Bloch vector of $1 \mathrm{D}$ silver/GaN stack with loss, but without gain. The green solid one is the result of stack with loss and gain. The black and blue solid curves are the same as those in Fig.2 (b), i.e. for lossless materials. The dotted vertical line illustrates the conservation of the parallel component of the wave vector. (b) Imaginary component part of the Bloch vector.

In Fig 5 (a), when losses are considered, the curve corresponding to the real component of Bloch vector (the red dashed one) is distorted compared with that in the lossless case (the blue solid one). The larger the angle of incidence (large value of $k_{x}$ ), the more distorted the curve. For the incident angle of $45^{\circ}$, the refracted angle deduced from the dispersion relation is $-38.7^{\circ}$ for the lossy structure.

In Fig. 5 (b), for propagating waves (i.e. $-1<k_{x} / k_{0}<1$ ), the imaginary part of the Bloch wave vector is null in lossless case while nonzero in lossy case. The nontrivial absorption decreases the transmission of the structure. 


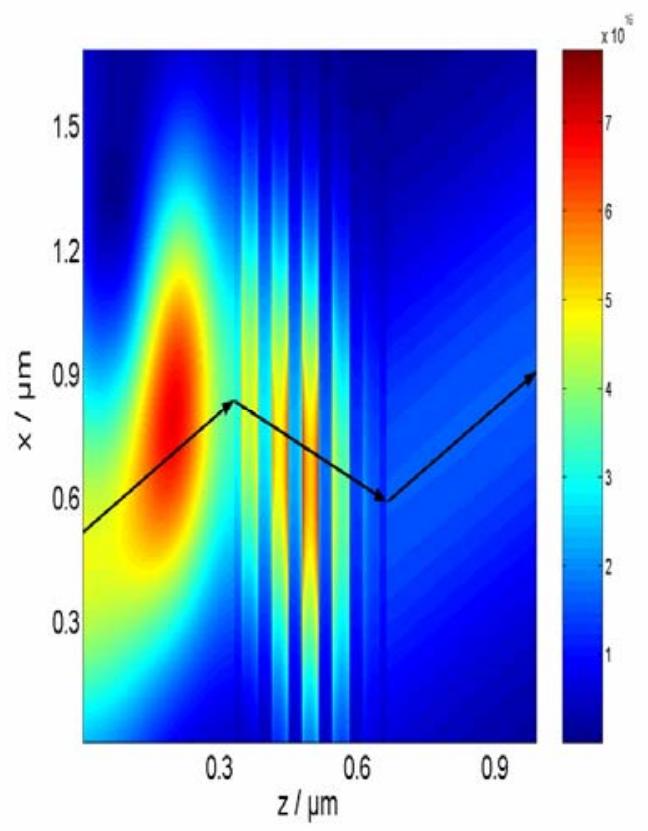

Fig.6. Identical to Fig.3 but with losses in the metal layers.

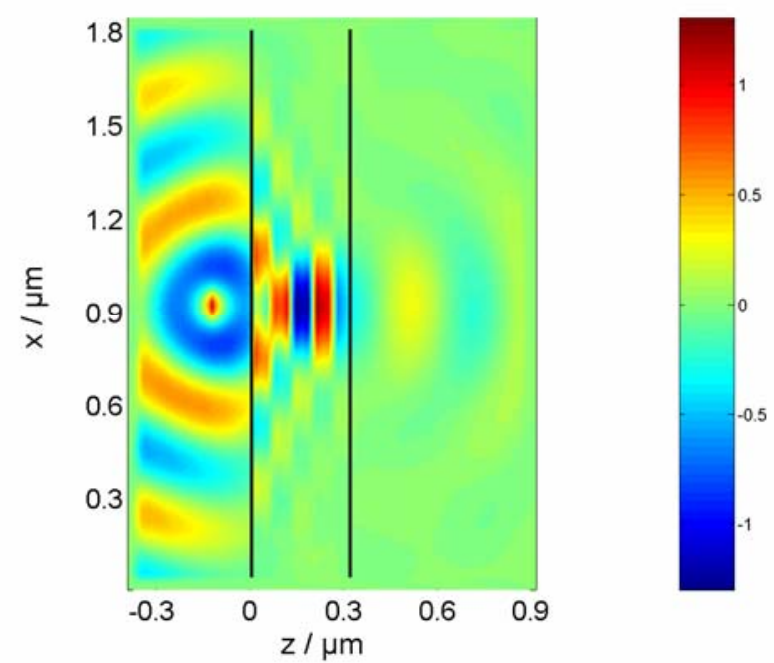

Fig.7. Identical to Fig.4 but with losses in the metal layers.

The time-averaged power density distribution in Fig. 6 confirms the influence of the losses. The refracted angle calculated according to the field distribution is about $-37.5^{\circ}$, in accordance with the $-38.7^{\circ}$ obtained in the dispersive curve. It is also seen from Fig. 6 that the transmittance is decreased obviously. The imaging process in the lossy case is shown in Fig. 7. Because of the decrease of the negative refraction angle and transmittance, the image is obscured. Moreover, the distance between image and object is $624 \mathrm{~nm}$, deviating the twice value of the slab width. The corresponding FWHM of the image is $0.78 \lambda$.

\section{Choose a gain-providing semiconductor to compensate for the losses in metals}

Since the absorption losses obviously influence the refraction angle and transmittance in the 1D stack, 
we try to use other structures containing gain-providing materials to compensate for losses and obtain a nearly isotropic -1 effective refractive index.

Recently Ramakrishna and Pendry suggested that gain-providing materials, e.g., semiconductor laser materials, stapled between the negative-index (or metal) layers of 1D stack [21] could compensate for the absorption effect and improve resolution. V. M. Shalaev and his co-authors showed that a composite material composed of silver strips and a gain-providing material can be perfectly transparent while still maintaining the negative refractive index [22]. Now we consider our stack with the dielectric medium replaced by a gain-providing medium (for example, a semiconductor laser material such as $\mathrm{GaN}$ or AlGaAs) to compensate for the losses in metal layers.

Using optical pumping one can make the GaN now optically amplifying in the UV region of the spectrum $(\lambda \approx 376 \mathrm{~nm})$ [23], where one can find suitable parameters to realize a nearly isotropic -1 effective index structure. By adjusting the intensity of pump laser, the imaginary part of the gain-providing medium can be tuned, at the operating wavelength, for example, $\varepsilon_{1}=\varepsilon_{\text {Gav }}=9-0.2 i$ [23]. The dispersion curve when gain is involved is shown in Fig.5 by the green solid curve. In the propagating wave region, the real part of the Bloch wave vector is almost the same as that in the lossless case [shown in Fig. 5(a)], and the imaginary one is reduced almost to zero [shown in Fig. 5(b)].

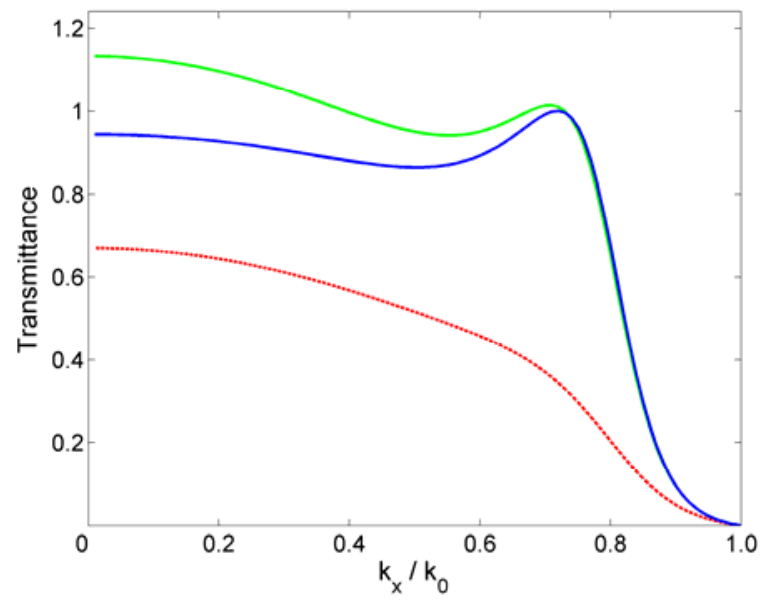

Fig.8. Transmittances of 5-period silver/GaN stacks. The blue line is for lossless case; the red dashed line is for a stack with loss, but without gain; the green line is for the stack with loss and gain.

Figure 8 shows the transmittances through the three different stacks with respect to the parallel wave vectors $k_{x}$. The blue line is for lossless case, the red dashed line is for a stack with loss, but without gain and the green line is for the stack with loss and gain. The transmittances of the lossless stack and the lossy stack with gain are almost unity in a wide range of $k_{x}$ compared with the low one of the lossy stack without gain.

The time-averaged power density distribution of 5-period silver/GaN stack with loss and gain for an incident angle of $45^{\circ}$ is given in Fig. 9. In the field map, the refraction angle is $-42.7^{\circ}$ comparable with the refraction angle from the dispersion curve $\left(-40.4^{\circ}\right)$ and the transmittance is 0.99 , nearing $-43.6^{\circ}$ and 0.98 in lossless case, respectively. 


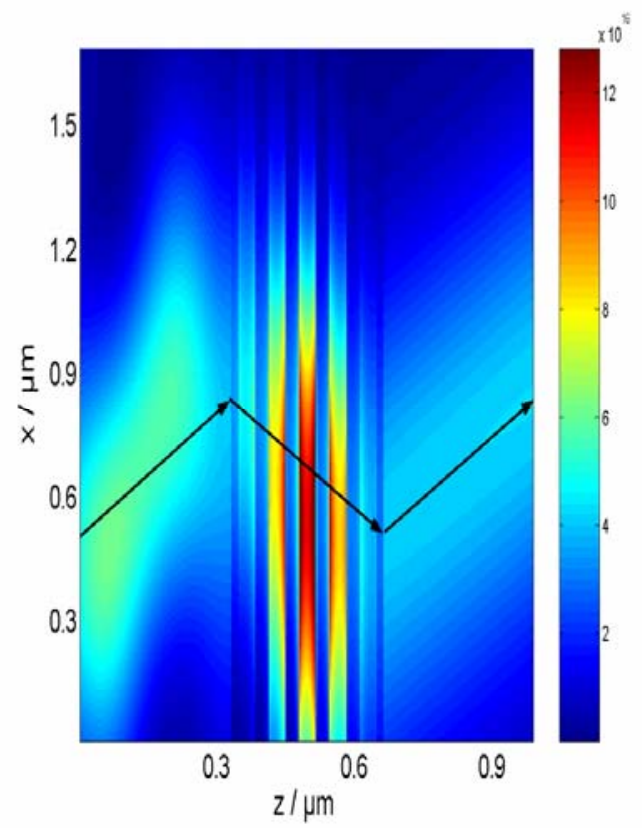

Fig.9. Time-averaged power density distribution of negative refraction with the 5-period silver/GaN stack with loss and gain as the angle of incidence is $45^{\circ}$.

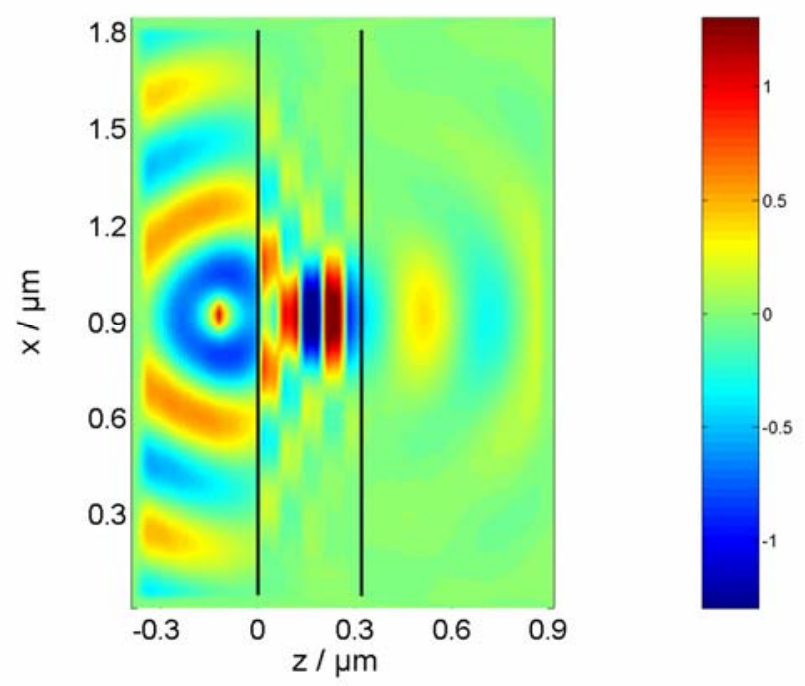

Fig.10. Magnetic distribution of imaging with the 5-period silver/GaN stack with loss and gain.

Figure 10 shows the imaging process with the 5-period silver/GaN stack. Because of the gain in GaN, the performance of the imaging is greatly improved, approaching the lossless case. The corresponding distance between image and object is about $633 \mathrm{~nm}$, and the FWHM is $0.64 \lambda$.

\section{Conclusion}

We have shown that nearly isotropic -1 effective index can be achieved using metal-dielectric stack containing gain-providing material. Considering realistic material parameters, we have studied the complex band structure and shown that the absorption losses in metallic layers not only decrease the transmittance, but also change the negative refraction angle in the stack (and thus its effective optical 
index). Then, we have proposed to use optical gain medium in 1D stack to compensate for the metallic losses and realize a near -1 effective index. In practice, a near -1 effective index stack with high transmittance can be realized using classical film deposition fabrication technology.

\section{Acknowledgement}

H. T. Jiang thanks La Fondation Franco-Chinoise pour la Science et ses Applications.

\section{References}

1. V. G. Veselago, "Electrodynamics of substances with simultaneously negative electrical and magnetic permeabilities" Sov. Phys. Usp. 10, 509-517 (1968).

2. J. B. Pendry, "Negative refraction makes a perfect lens," Phys. Rev. Lett. 85, 3966 (2000).

3. R. A. Shelby, D. Smith, and S. Schultz, "Experimental Verification of a Negative Index of Refraction," Science 292, $77-79$ (2001).

4. S. Linden, C. Enkrich, M. Wegener, J. Zhou, T. Koschny, and C. M. Soukoulis, "Magnetic response of metamaterials at 100 Terahertz" Science 306, 1351-1353 (2004).

5. B. Gralak, S. Enoch, and G. Tayeb, “Anomalous refractive properties of photonic crystals” J. Opt. Soc. Am. A 17, 1012-1020 (2000).

6. M. Notomi, "Theory of light propagation in strongly modulated photonic crystals: refractionlike behavior in the vicinity of the photonic band gap" Phys. Rev. B 62, 10696 (2000).

7. E. Cubukcu, K. Aydin, E. Ozbay, S. Foteinopoulou, and C. M. Soukoulis, "Electromagnetic waves: Negative refraction by photonic crystals" Nature (London) 423, 604-605 (2003).

8. D. O. Melville, R. J. Blaikie, "Super-resolution imaging through a planar silver layer", Optics Express 13, 2127-2134 (2005).

9. N. Fang, H. Lee, C. Sun, X. Zhang, "Sub-Diffraction-Limited Optical Imaging with a Silver Superlens", Science 308, 534-537 (2005).

10. T. Taubner, D. Korobkin, Y. Urzhumov, G. Shvets, R. Hillenbrand, "Near-Field Microscopy Through a SiC superlens", Science 313, 1595 (2006).

11. S. A. Ramakrishna, J. B. Pendry, M. C. K. Wiltshire, and W. J. Stewart, "Imaging the near field" J. Mod. Opt. 50, $1419-1430$ (2003).

12. Belov P A, Hao Yang, "Subwavelength imaging at optical frequencies using a transmission device formed by a period layered metal-dielectric structure operating in the canalization regime," Phys . Rev . B 73, 113110 (2006). 
13. H. Shin and S. H. Fan, "All-angle negative refraction and evanescent wave amplification using one-dimensional metallodielectric photonic crystals" Appl. Phys. Lett. 89, 151102 (2006).

14. M. Scalora, G. D'Aguanno, N. Mattiucci, M. J. Bloemer, D. de Ceglia, M. Centini, A. Mandatori, C. Sibilia, N. Akozbek, M. G. Cappeddu, M. Fowler, and J. W. Haus, "Negative refraction and sub-wavelength focusing in the visible range using transparent metallo-dielectric stacks" Opt. Express, 15, 508-523 (2007).

15. R. Pierre, and B. Gralak, "Appropriate truncation for photonic crystals", currently in production, Journal of Modern Optics.

16. J. L. Zhang, H. T. Jiang, B. Gralak, S. Enoch, G. Tayeb, and M. Lequime, “Towards -1 effective index with one-dimensional metal-dielectric metamaterial: a quantitative analysis of the role of absorption losses," Opt. Express 15, 7720 (2007)

17. P. Yeh, A. Yariv, and C. S. Hong, "Electromagnetic propagation in periodic stratified media. I . General theory," J. Opt. Soc. Am. 67, 423-438 (1977).

18. Kong J A, Wu B L, Zhang Y, “A unique lateral displacement of a Gaussian beam transmitted through a slab with negative permittivity and permeability,” Microwave Opt . Technol . Lett . 33, 136-139 (2002).

19. P. B. Johnson, R. W. Christy, “Optical constants of noble metals” Phys. Rev. B 6, 4370-4379 (1972).

20. V. Kuzmiak, A. A. Maradudin, "Photonic band structures of one- and two-dimensional periodic systems with metallic components in the presence of dissipation” Phys. Rev. B 55, 7427-7444 (1996).

21. S. A. Ramakrishna and J. B. Pendry, "Removal of absorption and increase in resolution in a near-field lens via optical gain,” Phys. Rev. B 67, 201101 (2003).

22. T. A. Klar, A. V. Kildishev, V. P. Drachev and V. M. Shalaev,"Negative-Index Metamaterials: Going Optical,” IEEE J. Selec. Top. Quant. Electron. 12, 1106-1115 (2006).

23. S. Strite and H. Morkoc, “GaN, AlN, and InN: A review,” J. Vac. Sci. Technol. B 10, 1237-1266 (1992). 